\title{
Evaluación de dos insecticidas naturales y un químico en el control de plagas en el cultivo de frejol en el litoral ecuatoriano
}

\author{
Evaluation of two natural insecticides and a chemical on pest control \\ in the crop of beans in the litoral ecuador \\ Rodrigo Paul Cabrera Verdezoto ${ }^{1-2 *}$ Jéssica Jessenia Morán Morán ${ }^{1-2}$, \\ Bladimir Juvencio Mora Velasquez, ${ }^{1}$ Humberto Manuel Molina Triviño ${ }^{1}$ \\ Oscar Fabián Moncayo Carreño ${ }^{1}$ Eduardo Díaz Ocampo 1 , \\ Gary Alex Meza Bone ${ }^{1-2}$, Cesar Alberto Cabrera Verdesoto ${ }^{3}$
}

\begin{abstract}
RESUMEN
La investigación se realizó en 2014 en la Finca Experimental "La María”, de la Universidad Técnica Estatal de Quevedo (UTEQ) localizada en el km 71/2 de la vía Quevedo - Mocache, Provincia de Los Ríos (0106’23” S y 79²9’ 12” W y 73 msnm). El objetivo del estudio fue evaluar la incidencia de insectos-plaga y su efecto sobre el rendimiento del cultivo de frejol (Phaseolus vulgaris $\mathrm{L}$.) como respuesta a la aplicación de insecticidas naturales durante el ciclo vegetativo. Se utilizaron formulaciones naturales a base de ají y tabaco en dosis de $10 \mathrm{~L} \mathrm{ha}^{-1}$ con tres frecuencias de aplicación: $\mathrm{A}_{1}=$ Dos aplicaciones ( $\left.15 \mathrm{dds}, 23 \mathrm{dds}\right), \mathrm{A}_{2}$ $=$ Tres aplicaciones $(15,23,31 \mathrm{dds})$ y $\mathrm{A}_{3}=$ Cuatro aplicaciones $(15,23,31$ y $44 \mathrm{dds})$; respectivamente. Las variables en estudio fueron: incidencia de insectos, eficacia de los tratamientos, números de vainas por planta, números de granos por vaina, peso de 100 semillas y rendimiento $\mathrm{ha}^{-1}$. Los resultados obtenidos muestran que las formulaciones a base de ají presentaron resultados similares al tratamiento químico en el control de mosca blanca, en relación al control de lorito verde, las formulaciones naturales redujeron la incidencia de lorito verde. El tratamiento T3 (ají + 3 aplicaciones) mostró mayor rendimiento en todas las variables evaluadas.

Palabras clave: ají, tabaco, monitoreo, mosca blanca, lorito verde.
\end{abstract}

\begin{abstract}
This research was conducted in 2014 at the Experimental Farm "La Maria" property of the State Technical University of Quevedo

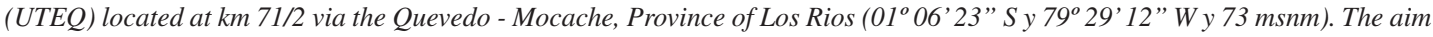
of the study was to evaluate the incidence of insect pests and its effect on crop yield of bean (Phaseolus vulgaris $L$.) in response to the application of natural insecticides during the growing cycle. Natural insecticides used were based aji and tobacco in doses of $10 \mathrm{~L} \mathrm{ha}^{-1}$ with three frequencies of application $A_{1}=$ Two applications $(15 \mathrm{dds}, 23 \mathrm{dds}), \mathrm{A}_{2}=$ Three applications $(15,23,31 \mathrm{dds})$ and $A_{3}=$ four applications $(15,23,31$ and $44 d d s)$ respectively. The variables studied were incidence of insect efficacy of treatments, number of pods per plant, grain number per pod, 100 seed weight and yield ha-1. The results show that the chili-based insecticide showed similar results to the chemical treatment to control whitefly, relative to control leafhopper, based insecticides chili and snuff showed better averages in monitoring 2 and 10 with the lower incidence of leafhopper. The productive variables it was determined in relation to the T3 (pepper + three applications) showed the highest averages in all variables.
\end{abstract}

Key words: chili, snuff, monitoring, whitefly, green parrot.

\section{Introducción}

Los estudios arqueológicos revelan que el frejol (Phaseolus vulgaris L.), se origina en el continente americano (Reyes, 2008). Existen evidencias de
8 mil años de antigüedad en algunas regiones de México y Perú (Voysest, 2000), que indican que fue un alimento básico muy importante en la dieta de los nativos americanos. Representa una de las principales fuentes alimentarias en Ecuador, ya que

\footnotetext{
Universidad Técnica Estatal de Quevedo, Facultad de Ciencias Pecuarias y Facultad de Ciencias Agrarias. Los Ríos (Ecuador). 2 Instituto Tecnológico Superior Ciudad de Valencia, Los Ríos (Ecuador).

3 Universidad Estatal del Sur de Manabí, Facultad de Ciencias Naturales y de la Agricultura. Manabí (Ecuador).

* Autor para correspondencia: rpcabrerav@uteq.edu.ec ing.rcabrerav@gmail.com
}

Fecha de Recepción: 11 Mayo, 2015.

Fecha de Aceptación: 25 Agosto, 2016.

DOI: $10.4067 /$ S0718-34292016005000025 
posee un alto contenido de proteínas (más del 20\%) y otros nutrientes que son necesarios para la dieta diaria (Reyes, 2008). Sin embargo, el consumo per cápita del mismo es de 2,6 kg (persona año ${ }^{-1}$ ), inferior al consumo de otros países de la región andina, tal como Bolivia, cuyo consumo es $10 \mathrm{~kg}$ (persona año-1) (Valladolid et al., 1998).

En Ecuador es uno de los alimentos de mayor consumo, debido a su valor accesible, contenido de grasas y proteínas, comparable en valor nutritivo con el de la carne roja (Garcés, 2013), siendo consumida tanto como grano seco y/o como vaina verde (Ernest et al., 2008).

La superficie promedio cultivada en Ecuador en los últimos cinco años es de $22.186 \mathrm{ha}^{-1}$, correspondientes a $3.410 \mathrm{ha}^{-1}$ para frejol arbustivo (hábito de crecimiento determinado) y 18.776 ha $^{-1}$ para voluble (hábito de crecimiento indeterminado). En el mismo período, el rendimiento promedio para frejol seco fue de $350 \mathrm{~kg} \mathrm{ha}^{-1} \mathrm{y}$ para vaina verde de $1.340 \mathrm{~kg} \mathrm{ha}^{-1}$ (INEC, 2002). El cultivo de esta leguminosa lo desarrollan pequeños agricultores, orientando la producción al autoconsumo, $\mathrm{y}$ al abastecimiento del mercado interno. Se siembra principalmente en el litoral ecuatoriano preferiblemente en la época de verano, con variedades de color claro: amarillo (canario), blanco (caballero) y rojo, siendo influenciada por la región y gusto del consumidor (Garcés et al., 2015).

Debido a la intensa aplicación de insumos para el control de plagas y/o fertilizantes sintéticos, los suelos se han deteriorado porque los fertilizantes químicos son de acción inmediata y, al mismo tiempo, solo funcionan al corto plazo, produciendo un desbalance en la agricultura y también un desequilibrio ambiental (Altieri y Nicholls, 2000) asimismo, potencia la aparición de nuevas plagas y de un desgaste del suelo o la resistencia de estas a los insumos químicos, ocasionando una dependencia cada vez mayor a la industria agroquímica (Pérez y Landeros, 2009).

Con el propósito de reducir el uso de insecticidas sintéticos y a la vez apoyar el control de insectosplaga, se plantea la búsqueda de productos de origen vegetal que tengan efecto insecticida y resulten saludables, eficientes y ecológicamente aceptables, lo cual constituye una forma de retorno a una práctica agrícola tradicional. $\mathrm{Al}$ ser estos compuestos de naturaleza biodegradable, ofrecen seguridad relativa a organismos benéficos, reduciendo las poblaciones de insectos-plaga resistentes a los agroquímicos (Maggi, 2004). En este contexto, extracto de varias plantas han sido utilizadas por su efecto insecticida, entre ellas el ají planta del género Capsicum, cuyo principio activo es la capsaicina ha sido ampliamente utilizadas para el control de insectos minadores, chupadores, barrenadores y masticadores. Presenta acción repelente y actúa por ingestión, causando trastornos digestivos, por lo tanto, el insecto deja de alimentarse. Su principio insecticida se encuentra distribuido principalmente en el fruto, siendo está la parte más comúnmente utilizada (Madhumathy et al., 2007; Peña et al., 2013). Por su parte el tabaco, es utilizado en agricultura orgánica gracias a su acción fungicida, insecticida, repelente y acaricia; propiedades atribuidas a su principal componente; la nicotina que actúa como una sustancia tóxica de contacto e ingestión; y se ha formulado como sulfato de nicotina (Casanova et al., 2002; Fuentes et al., 2007; Murillo et al., 2012).

El alto costo de los insecticidas químicos y en ocasiones su baja disponibilidad los hace poco accesibles a los pequeños agricultores, lo cual limita el control oportuno, traduciéndose en pérdida del rendimiento (Horwith, 1985; Altieri, 1999), además de los efectos adversos que estos provocan en el ambiente alterando el equilibrio natural del ecosistema, ya que pueden llegar a contaminarlas además de crear resistencia de los insectos y afectar la salud humana (Cardona et al., 2005).

El objetivo del estudio fue evaluar la incidencia de insectos-plaga y su efecto sobre el rendimiento el cultivo de frejol (Phaseolus vulgaris L.) como respuesta a la aplicación de insecticidas naturales.

\section{Materiales y Métodos}

La investigación se realizó durante la época de verano (junio-septiembre) del 2014, en la Finca Experimental "La María", propiedad de la Universidad Técnica Estatal de Quevedo, localizada en $01^{\circ} 06^{\prime} 23^{\prime \prime} \mathrm{S}$ y $79^{\circ} 29^{\prime} 12^{\prime \prime}$ W y $73 \mathrm{msnm}$. El clima se caracteriza como bosque húmedo tropical (bh-T) con topografía plana, suelo de textura francoarcillosa, tipo Inceptisoles y $\mathrm{pH}$ 5,8 caracterizado por una temperatura media diaria de $24,2{ }^{\circ} \mathrm{C}$, precipitación anual promedio de 1.537 $\mathrm{mm}$, una humedad relativa $77,4 \%$ y 823 horas sol al año (División de Meteorología, Departamento de Sinóptica del Instituto Nacional Autónomo de Investigaciones Agropecuarias (INIAP), Quevedo). 
Se trabajó con frejol tipo arbustivo, variedad EVG-6, cuyas características agronómicas son: granos color marrón, floración (siembra a la floración) 29-32 días; maduración desde la siembra a la cosecha 66 días; altura de planta, $34-37 \mathrm{~cm}$; 10 vainas por planta; 5 semillas por vaina; peso de 100 semillas, $55-88 \mathrm{~g}$; rendimiento 1.110 a $1.115 \mathrm{~kg} \mathrm{ha}^{-1}$.

\section{Diseño experimental}

Las fuentes de variación correspondieron a:

\section{Insecticida natural:}

En base a ají $\left(10 \mathrm{~L} \mathrm{ha}^{-1}\right)$. En base a tabaco $\left(10 \mathrm{~L} \mathrm{ha}^{-1}\right)$.

2. Número de $A_{1}=$ Dos aplicaciones ( 15 y 23 días). aplicaciones: $\mathrm{A}_{2}=$ Tresaplicaciones (15,23y 31 días). $\mathrm{A}_{3}=$ Cuatro aplicaciones $(15,23$, 31 y 44 días).

El diseño experimental fue de bloques completos al azar en arreglo factorial $2 \times 3$, definiendo 6 tratamientos correspondientes a la combinación de los factores experimentales, a los que se agregó un control químico y un control absoluto en cuatro repeticiones por cada tratamiento, haciendo un total de 32 unidades experimentales (Tabla 1).

Los datos se sometieron a pruebas de normalidad, homogeneidad de varianzas con el test de ShapiroWilk, y prueba de contraste de medias con el test de Tukey al 95\% (P>0,05). Para el análisis se trabajó con el programa Statgrafic Centurión XV.

\section{Manejo experimental}

La preparación de suelo consistió en laboreo con rastra de disco, tres días antes de la siembra. Antes del rastraje el suelo se regó con una carga de agua equivalente a $5 \mathrm{~L} \mathrm{~m}^{2}$. La siembra se hizo el 10 de junio del 2014, utilizando un espeque a una

Tabla 1. Tratamientos implementados en la evaluación de campo.

\begin{tabular}{cl}
\hline Código & \multicolumn{1}{c}{ Detalle } \\
\hline T0 & Control absoluto (-) \\
T1 & Control químico (+) clorpirifós \\
T2 & Extracto ají en dos aplicaciones \\
T3 & Extracto ají en tres aplicaciones \\
T4 & Extracto ají en cuatro aplicaciones \\
T5 & Extracto tabaco en dos aplicaciones \\
T6 & Extracto tabaco en tres aplicaciones \\
T7 & Extracto tabaco en cuatro aplicaciones \\
\hline
\end{tabular}

profundidad de $2,5 \mathrm{~cm}$, depositando una semilla por golpe. Se establecieron parcelas de cinco hileras de $4 \mathrm{~m}$ de longitud como unidad experimental. La distancia entre hileras fue de $0,50 \mathrm{~m}$ y la distancia entre planta fue de $0,20 \mathrm{~cm}$, conformando parcelas de $8 \mathrm{~m}^{2}$ con un área experimental total de $600 \mathrm{~m}^{2}$. Antes de la siembra, las semillas se trataron con Carboxin (Vitavax ${ }^{\circledR}$ ) en dosis de $1,5 \mathrm{~kg} \mathrm{ha}^{-1}$. Se fertilizó de forma fraccionada a los 8 y 20 (dds) días después de la siembra a razón de $80 \mathrm{~kg} \mathrm{~N}$ $\mathrm{ha}^{-1}$ (Urea), $40 \mathrm{~kg} \mathrm{P}_{2} \mathrm{O}_{5} \mathrm{ha}^{-1}$ (Súperfosfato triple) y $40 \mathrm{~kg} \mathrm{~K}_{2} \mathrm{O} \mathrm{ha}^{-1}$ (Muriato de potasio), mientras el $50 \%$ restante se aplicó a los 20 días, la fertilización se hizo con un espeque a una distancia de $5 \mathrm{~cm}$ de la planta. La irrigación se la realizó en función de la necesidad del cultivo. La cosecha se realizó el 25 de agosto de 2014 ( 75 dds) correspondiendo a la maduración fisiológica-vainas totalmente secas (Hall, 1994). Para el control de malezas se aplicó pendimetalina en preemergencia, con dosis de 1,5 $\mathrm{L} \mathrm{ha}^{-1}$. Se complementó con dos escardas manuales durante el desarrollo del cultivo. Para el control de insectos chupadores y gusanos cortadores se aplicó los tratamientos definidos en el diseño experimental (ají y tabaco), El control químico correspondió al insecticida Delta cloro (clorpirifós) en dosis $1 \mathrm{~L} \mathrm{ha}^{-1}$. Esto fueron aplicados e diferentes frecuencias lo que consiste en el número que se aplicó los diferentes insecticidas durante la investigación.

\section{Incidencia de insectos}

Para determinar la población de mosca blanca (Bemisia tabaci) y lorito verde (Empoasca kraemeri Ross y Moore) se hicieron 12 muestreos, escogiendo cinco plantas al azar dentro cada parcela un día antes, y uno y cuatro días después de la aplicación de los tratamientos. La población de adultos presentes se determinó en el folíolo central de la hoja clave (tercera hoja superior) de cada una de las plantas previamente seleccionadas en las cuales se realizó conteo directo, considerándose como población inicial el número de individuos registrados en el muestreo un día antes de la primera aplicación (Tablas 2 y 3 ). La eficacia de los tratamientos se determinó en base al número de insectos en el testigo con respecto a los demás tratamientos, lo cual fue expresado en porcentaje, por medio de la fórmula de eficacia (Abbott, 1925):

$$
\text { Eficacia } \%=\frac{\mathrm{ICt}-\mathrm{IVT}}{\mathrm{IVt} * 100}
$$


Tabla 2. Efecto de 8 tratamientos en la población adulta de mosca blanca (Bemisia tabaci) en 12 muestreos sistemáticos.

\begin{tabular}{|c|c|c|c|c|c|c|c|c|c|c|c|c|}
\hline \multirow{2}{*}{ Tratamiento } & \multicolumn{12}{|c|}{ Muestreo } \\
\hline & 1 & 2 & 3 & 4 & 5 & 6 & 7 & 8 & 9 & 10 & 11 & 12 \\
\hline Ají & $2,17 \mathrm{a}$ & $1,37 \mathrm{~b}$ & $1,30 \mathrm{~b}$ & $1,68 \mathrm{~b}$ & $1,25 \mathrm{~b}$ & $1,25 \mathrm{~b}$ & $1,69 \mathrm{a}$ & $1,34 \mathrm{~b}$ & $1,43 \mathrm{a}$ & $1,63 \mathrm{a}$ & $1,58 \mathrm{a}$ & $1,57 \mathrm{a}$ \\
\hline Tabaco & 2,16 a & $1,49 \mathrm{a}$ & $1,45 \mathrm{a}$ & $1,79 \mathrm{a}$ & $1,36 \mathrm{a}$ & $1,40 \mathrm{a}$ & $1,68 \mathrm{a}$ & $1,42 \mathrm{a}$ & $1,46 \mathrm{a}$ & $1,56 \mathrm{a}$ & $1,49 \mathrm{~b}$ & $1,47 \mathrm{a}$ \\
\hline \multicolumn{13}{|l|}{ Frecuencias $(\mathbf{F})$} \\
\hline $\mathrm{F}_{1}=2$ aplicaciones & $2,08 \mathrm{a}$ & $1,41 \mathrm{a}$ & $1,34 \mathrm{a}$ & $1,75 \mathrm{a}$ & $1,31 \mathrm{a}$ & $1,32 \mathrm{a}$ & $1,70 \mathrm{a}$ & $0,90 \mathrm{a}$ & $1,77 \mathrm{a}$ & $1,74 \mathrm{a}$ & $1,77 \mathrm{a}$ & $1,66 \mathrm{a}$ \\
\hline $\mathrm{F}_{2}=3$ aplicaciones & $2,19 \mathrm{a}$ & $1,48 \mathrm{a}$ & $1,41 \mathrm{a}$ & $1,71 \mathrm{a}$ & $1,27 \mathrm{a}$ & $1,36 \mathrm{a}$ & $1,72 \mathrm{a}$ & $0,92 \mathrm{a}$ & $1,30 \mathrm{~b}$ & $1,54 \mathrm{~b}$ & $1,56 \mathrm{~b}$ & $1,62 \mathrm{a}$ \\
\hline $\mathrm{F}_{3}=4$ aplicaciones & $2,23 \mathrm{a}$ & $1,40 \mathrm{a}$ & 1,39 a & $1,76 \mathrm{a}$ & $1,34 \mathrm{a}$ & $1,30 \mathrm{a}$ & $1,63 \mathrm{a}$ & $0,89 \mathrm{a}$ & $1,27 \mathrm{~b}$ & $1,51 \mathrm{~b}$ & $1,29 \mathrm{c}$ & $1,27 \mathrm{~b}$ \\
\hline \multicolumn{13}{|l|}{ Tratamientos } \\
\hline T0 Testigo absoluto & $2,12 \mathrm{a}$ & $1,80 \mathrm{a}$ & $1,88 \mathrm{a}$ & $1,99 \mathrm{a}$ & $1,84 \mathrm{a}$ & $1,76 \mathrm{a}$ & $1,82 \mathrm{a}$ & $1,80 \mathrm{a}$ & $1,77 \mathrm{a}$ & $1,82 \mathrm{a}$ & $1,78 \mathrm{a}$ & $1,73 \mathrm{a}$ \\
\hline T1 Química (Clorpirifós) & $2,63 \mathrm{a}$ & $1,43 \mathrm{bc}$ & $1,26 \mathrm{~b}$ & $1,64 b$ & $1,24 \mathrm{bc}$ & $1,30 \mathrm{~b}$ & $1,47 \mathrm{c}$ & $1,20 \mathrm{~d}$ & $1,22 \mathrm{c}$ & $1,46 \mathrm{c}$ & 1,37 ed & $1,34 \mathrm{bc}$ \\
\hline T2 (Ají + 2 aplicaciones) & $2,06 \mathrm{a}$ & $1,41 \mathrm{bc}$ & $1,28 \mathrm{~b}$ & $1,67 \mathrm{~b}$ & $1,22 \mathrm{c}$ & $1,24 \mathrm{~b}$ & $1,67 \mathrm{ab}$ & $1,61 \mathrm{~b}$ & $1,73 \mathrm{a}$ & $1,76 \mathrm{ab}$ & $1,78 \mathrm{a}$ & $1,66 \mathrm{a}$ \\
\hline T3 (Ají + 3 aplicaciones) & $2,28 \mathrm{a}$ & $1,34 \mathrm{c}$ & $1,34 \mathrm{~b}$ & $1,66 \mathrm{~b}$ & $1,22 \mathrm{c}$ & $1,28 \mathrm{~b}$ & $1,76 \mathrm{ab}$ & $1,20 \mathrm{~d}$ & $1,28 \mathrm{bc}$ & $1,61 \mathrm{bc}$ & $1,61 \mathrm{bc}$ & $1,70 \mathrm{a}$ \\
\hline T4 (Ají + 4 aplicaciones) & $2,17 \mathrm{a}$ & $1,39 \mathrm{bc}$ & $1,28 \mathrm{~b}$ & $1,73 \mathrm{~b}$ & $1,32 \mathrm{bc}$ & $1,24 \mathrm{~b}$ & $1,64 \mathrm{bc}$ & $1,20 \mathrm{~d}$ & $1,28 \mathrm{bc}$ & $1,53 \mathrm{c}$ & 1,35 ef & $1,35 \mathrm{bc}$ \\
\hline T5 (Tabaco + 2 aplicaciones) & $2,09 \mathrm{a}$ & $1,44 \mathrm{bc}$ & $1,39 \mathrm{~b}$ & $1,83 \mathrm{ab}$ & $1,40 \mathrm{~b}$ & $1,41 \mathrm{~b}$ & $1,73 \mathrm{ab}$ & $1,61 \mathrm{~b}$ & $1,80 \mathrm{a}$ & $1,72 \mathrm{ab}$ & $1,75 \mathrm{ab}$ & $1,67 \mathrm{a}$ \\
\hline T6 (Tabaco + 3 aplicaciones) & $2,09 \mathrm{a}$ & $1,63 \mathrm{ab}$ & $1,47 \mathrm{~b}$ & $1,76 \mathrm{~b}$ & $1,32 \mathrm{bc}$ & $1,44 \mathrm{ab}$ & $1,67 \mathrm{ab}$ & $1,30 \mathrm{~cd}$ & $1,32 \mathrm{~b}$ & $1,48 \mathrm{c}$ & $1,51 \mathrm{~cd}$ & $1,55 \mathrm{ab}$ \\
\hline T7 (Tabaco + 4 aplicaciones) & $2,29 \mathrm{a}$ & $1,43 b c$ & $1,49 \mathrm{~b}$ & $1,79 a b$ & $1,36 \mathrm{bc}$ & $1,36 \mathrm{~b}$ & $1,62 b c$ & $1,34 \mathrm{c}$ & $1,26 \mathrm{bc}$ & $1,50 \mathrm{c}$ & $1,22 \mathrm{f}$ & $1,20 \mathrm{c}$ \\
\hline C.V. $\%$ & 13,43 & 7,73 & 7,02 & 5,51 & 5,19 & 10,21 & 4,51 & 4,09 & 2,77 & 4,12 & 4,12 & 7,09 \\
\hline
\end{tabular}

Promedios con letras iguales dentro de cada factor no presentan diferencias estadísticas según $\mathrm{F}(\mathrm{P}>0,05)$.

Tabla 3. Efecto de 8 tratamientos en la población adulta de lorito verde

(Empoasca kraemeri Ross y Moore) en 12 muestreos sistemáticos.

\begin{tabular}{|c|c|c|c|c|c|c|c|c|c|c|c|c|}
\hline \multirow{2}{*}{ Tratamientos } & \multicolumn{12}{|c|}{ Muestreo } \\
\hline & 1 & 2 & 3 & 4 & 5 & 6 & 7 & 8 & 9 & 10 & 11 & 12 \\
\hline Ají & $1,50 \mathrm{a}$ & $1,41 \mathrm{~b}$ & $1,26 \mathrm{a}$ & $1,40 \mathrm{a}$ & $1,25 \mathrm{a}$ & $1,29 \mathrm{a}$ & $1,32 \mathrm{a}$ & $1,34 \mathrm{a}$ & $1,37 \mathrm{a}$ & $1,42 \mathrm{a}$ & $1,31 \mathrm{a}$ & $1,35 \mathrm{a}$ \\
\hline Tabaco & $1,43 \mathrm{a}$ & $1,43 \mathrm{a}$ & $1,25 \mathrm{a}$ & $1,43 \mathrm{a}$ & $1,29 \mathrm{a}$ & $1,36 \mathrm{a}$ & $1,36 \mathrm{a}$ & $1,35 \mathrm{a}$ & $1,34 \mathrm{a}$ & $1,36 \mathrm{~b}$ & $1,29 \mathrm{a}$ & $1,32 \mathrm{a}$ \\
\hline \multicolumn{13}{|l|}{ Frecuencias (F) } \\
\hline $\mathrm{F}_{1}=2$ aplicaciones & $1,46 \mathrm{a}$ & $1,42 \mathrm{ab}$ & $1,22 \mathrm{a}$ & $1,36 \mathrm{a}$ & $1,22 \mathrm{a}$ & $1,30 \mathrm{a}$ & $1,37 \mathrm{a}$ & $1,38 \mathrm{a}$ & $1,39 \mathrm{a}$ & $1,40 \mathrm{a}$ & $1,36 \mathrm{a}$ & $1,40 \mathrm{a}$ \\
\hline $\mathrm{F}_{2}=3$ aplicaciones & $1,47 \mathrm{a}$ & $1,43 \mathrm{a}$ & $1,25 \mathrm{a}$ & $1,47 \mathrm{a}$ & $1,29 \mathrm{a}$ & $1,35 \mathrm{a}$ & $1,30 \mathrm{a}$ & $1,33 \mathrm{a}$ & $1,34 \mathrm{a}$ & $1,37 \mathrm{a}$ & $1,30 \mathrm{ab}$ & $1,35 \mathrm{ab}$ \\
\hline $\mathrm{F}_{3}=4$ aplicaciones & $1,47 \mathrm{a}$ & $1,41 \mathrm{~b}$ & $1,30 \mathrm{a}$ & $1,41 \mathrm{a}$ & $1,29 \mathrm{a}$ & $1,33 \mathrm{a}$ & $1,36 \mathrm{a}$ & $1,33 \mathrm{a}$ & $1,34 \mathrm{a}$ & $1,40 \mathrm{a}$ & $1,25 \mathrm{~b}$ & $1,27 \mathrm{~b}$ \\
\hline \multicolumn{13}{|l|}{ Tratamientos } \\
\hline T0 Testigo absoluto & $1,39 \mathrm{a}$ & $1,36 \mathrm{a}$ & $1,46 \mathrm{a}$ & $1,46 \mathrm{a}$ & $1,36 \mathrm{a}$ & $1,44 \mathrm{a}$ & $1,35 \mathrm{a}$ & $1,41 \mathrm{a}$ & $1,36 \mathrm{a}$ & $1,39 \mathrm{a}$ & $1,45 \mathrm{a}$ & $1,37 a b$ \\
\hline T1 Química (Clorpirifós) & $1,34 \mathrm{a}$ & $1,18 \mathrm{ab}$ & $1,07 \mathrm{~b}$ & $1,47 \mathrm{a}$ & $1,24 \mathrm{a}$ & $1,39 \mathrm{a}$ & $1,32 \mathrm{a}$ & $1,22 \mathrm{ab}$ & $1,18 \mathrm{~b}$ & $1,36 \mathrm{ab}$ & $1,24 \mathrm{bc}$ & $1,39 a b$ \\
\hline T2 (Ají + 2 aplicaciones) & $1,53 \mathrm{a}$ & $1,23 \mathrm{ab}$ & $1,26 \mathrm{ab}$ & $1,34 \mathrm{a}$ & $1,22 \mathrm{a}$ & $1,26 \mathrm{a}$ & $1,32 \mathrm{a}$ & $1,39 a$ & $1,41 \mathrm{a}$ & $1,39 \mathrm{ab}$ & $1,39 \mathrm{ab}$ & $1,41 \mathrm{a}$ \\
\hline T3 (Ají + 3 aplicaciones) & $1,48 \mathrm{a}$ & $1,26 a b$ & $1,20 \mathrm{ab}$ & $1,47 \mathrm{a}$ & $1,24 \mathrm{a}$ & $1,35 \mathrm{a}$ & $1,32 \mathrm{a}$ & $1,34 \mathrm{ab}$ & $1,35 \mathrm{a}$ & $1,38 a b$ & $1,26 \mathrm{bc}$ & $1,35 a b$ \\
\hline T4 (Ají + 4 aplicaciones) & $1,48 \mathrm{a}$ & $1,14 \mathrm{~b}$ & $1,33 \mathrm{ab}$ & $1,38 \mathrm{a}$ & $1,28 \mathrm{a}$ & $1,26 \mathrm{a}$ & $1,33 \mathrm{a}$ & $1,30 \mathrm{ab}$ & $1,35 \mathrm{a}$ & $1,50 \mathrm{a}$ & $1,28 \mathrm{bc}$ & $1,30 a b$ \\
\hline T5 (Tabaco + 2 aplicaciones) & $1,39 \mathrm{a}$ & $1,24 \mathrm{ab}$ & $1,18 \mathrm{~b}$ & $1,39 \mathrm{a}$ & $1,22 \mathrm{a}$ & $1,33 \mathrm{a}$ & $1,42 \mathrm{a}$ & $1,37 \mathrm{ab}$ & $1,37 \mathrm{a}$ & $1,41 \mathrm{ab}$ & $1,32 \mathrm{abc}$ & $1,39 a b$ \\
\hline T6 (Tabaco + 3 aplicaciones) & $1,45 \mathrm{a}$ & $1,32 \mathrm{ab}$ & $1,29 \mathrm{ab}$ & $1,46 \mathrm{a}$ & $1,34 \mathrm{a}$ & $1,35 \mathrm{a}$ & $1,28 \mathrm{a}$ & $1,32 \mathrm{ab}$ & $1,32 \mathrm{ab}$ & $1,36 \mathrm{ab}$ & $1,34 \mathrm{abc}$ & $1,35 a b$ \\
\hline T7 (Tabaco + 4 aplicaciones) & $1,45 \mathrm{a}$ & $1,20 \mathrm{ab}$ & $1,27 \mathrm{ab}$ & $1,44 \mathrm{a}$ & $1,30 \mathrm{a}$ & $1,40 \mathrm{a}$ & $1,38 \mathrm{a}$ & $1,35 \mathrm{ab}$ & $1,32 \mathrm{ab}$ & $1,30 \mathrm{~b}$ & $1,22 \mathrm{c}$ & $1,24 \mathrm{~b}$ \\
\hline C.V. $\%$ & 9,72 & 6,76 & 8,99 & 6,48 & 6,40 & 9,84 & 7,28 & 5,10 & 4,74 & 4,71 & 5,25 & 4,79 \\
\hline
\end{tabular}

Promedios con letras iguales dentro de cada factor no presentan diferencias estadísticas según $\mathrm{F}(\mathrm{P}>0,05)$.

\section{Variable productiva}

El número de vainas por planta se determinó contando el total de vainas presentes en 10 plantas tomadas al azar dentro del área útil de cada parcela.
Asimismo, el número de semillas por vaina se evaluó en las mismas plantas en las que se registró el número de vainas por planta, contabilizando el número total de granos. Para el peso de 100 semillas (g) se procedió a contar 100 granos de cada parcela útil, 
los mismos que fueron pesados en balanza digital (Ohaus ${ }^{\circledR}$ Scout Pro Balance, SPS2001F, capacidad $2000 \mathrm{~g}$, USA). Finalmente el rendimiento por $\mathrm{ha}^{-1}$ se determinó en base al peso total de los granos cosechados en cada parcela útil. El peso del grano se ajustó al $13 \%$ de humedad y se expresó en $\mathrm{kg}$ $\mathrm{ha}^{-1}$, mediante la siguiente fórmula:

$$
\operatorname{Pf}=\frac{\operatorname{Pi}(100-\mathrm{Hi})}{(100-\mathrm{Hf})}
$$

Donde:

$\mathrm{Pf}=$ Peso final $(\mathrm{kg} / \mathrm{ha})$.

$\mathrm{Hf}=$ Humedad final a la que se desea ajustar el rendimiento $(13 \%)$.

$\mathrm{Pi}=$ Peso inicial de campo $(\mathrm{Kg} / \mathrm{ha})$.

$\mathrm{Hi}=$ Humedad inicial.

\section{Resultados y Discusión}

\section{Incidencia de mosca blanca (Bemisia tabaci)}

La población de Bemisia tabaci, un día antes, uno y cuatro días después de la aplicación de los tratamientos en los 12 muestreos se presenta en la Tabla 2. La población presentó diferencias estadísticas altamente significativas entre los tratamientos. El insecticida a base de ají presentó mejor promedio en los muestreos 2, 3, 4, 5, 6 y 8 , con menor incidencia, difiriendo estadísticamente de la aplicación de insecticidas a base de tabaco que registró mayor incidencia de mosca blanca con incidencia entre 1,36 a 2,16 individuos por planta (Tabla 2). Se observaron diferencias estadísticas en los muestreos 9, 10, 11 y 12, mostrando mayor incidencia en la aplicación 1, mientras la menor incidencia se obtuvo en la aplicación 3 al muestreo 9 con 1,29; el T0 presentó las mayores incidencias de mosca blanca en todo los muestreos, mientras que los insecticidas naturales a base de ají y tabaco en los diferentes días de aplicación mantuvieron igualdad estadística al T1, el insecticida natural a base de ají mostró mejor comportamiento en las tres frecuencias de aplicación T2 (ají + 2 aplicaciones), T3 (ají +3 aplicaciones) y T4 (ají +4 aplicaciones); respectivamente (Tabla 2), lo cual concuerda con lo expuesto por Karlsson (2005), quien determinó que un insecticida a base de jabón de coco $3,5 \mathrm{~g} \mathrm{~L}^{-1}$ + ají $10 \mathrm{~g} \mathrm{~L}^{-1}$, mostró los mejores resultados en un cultivo de yuca. Asimismo, Firester (2011), indica que utilizando tres tipos de extractos vegetales: ají, ajo y abeto oriental, el ají manifestó una efectividad del $95 \%$ en el control de insectos, a su vez el insecticida a base de tabaco mostró menor incidencia de mosca blanca en los tratamientos con mayor frecuencia de aplicación como T6 (tabaco + 3 aplicaciones) y T7 (tabaco +4 aplicaciones) en todos los muestreos. Estos resultados concuerdan con lo mencionado por Manzano y Mejia (1998), quienes determinaron que el extracto de tabaco mantuvo mejor comportamiento en el control de plagas en el cultivo de frejol, con 14,20 moscas blancas por hoja, en relación a los demás extractos utilizados (ortiga y ajenjo) que obtuvieron 25,70 y 31,54 respectivamente.

\section{Incidencia de lorito verde (Empoasca kraemeri Ross y Moore)}

La Tabla 3 muestra el efecto de los tratamientos en la incidencia de lorito verde un día antes, un día después y cuatro días después de la aplicación de los insecticidas naturales para 12 evaluaciones. Los insecticidas a base de ají y tabaco presentaron el mejor promedios en los monitoreos 2 y 10 con la menor incidencia de lorito verde, evidenciando alta significancia estadística. Los insecticidas naturales aplicados en 3 aplicaciones en las evaluaciones 2, 11 y 12 presentaron menor incidencia de lorito verde, difiriendo estadísticamente de las demás aplicaciones. Entre los tratamientos se observó diferencias altamente significativas siendo T0 (control absoluto) el que presentó la mayor incidencia de lorito verde en todos los monitoreos realizados, destacando el comportamiento que mantuvieron los insecticidas a base de ají y tabaco en siete de los doce monitoreos ya que mostraron baja incidencia de lorito verde en las tres frecuencias de aplicación, al igual que el tratamiento T1 (clorpirifós) en dosis de $1 \mathrm{~L} \mathrm{ha}^{-1}$ (Tabla 3 ). Estos resultados no coinciden con los mencionados por Mendoza (1997), quien indica que el uso de los extractos vegetales evaluados en frejol en Honduras para el control de ninfas y adultos de lorito verde, no fue satisfactorio, siendo el insecticida químico Thiodan el que mostró menor incidencia en el control de lorito verde.

\section{Eficacia de los Tratamientos}

\section{Mosca blanca (Bemisia tabacci)}

La Figura 1 presenta la eficacia de los insecticidas, sobre la incidencia de mosca blanca 1 día después 


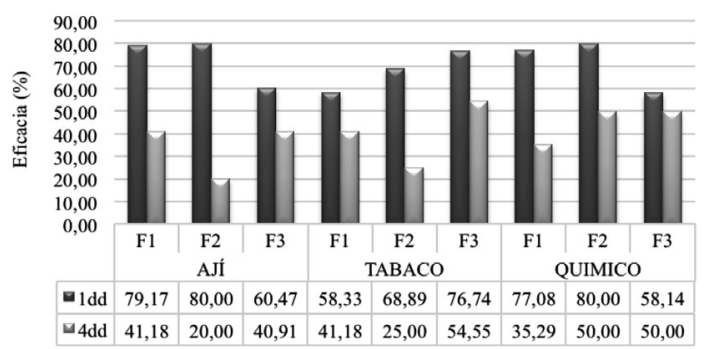

Figura 1. Eficacia de 9 tratamientos en los muestreos efectuados 1 día después y 4 días después de cada frecuencia de aplicación, sobre la población de mosca blanca (Bemisia tabacci).

de la segunda aplicación (F1). El tratamiento con mayor eficacia fue el insecticida natural a base de ají con 79,17\% seguido del tratamiento químico (clorpirifós) y el tabaco con 77,08 y $58,33 \%$ respectivamente; una vez transcurridos 4 días de aplicación fueron los insecticidas naturales a base de ají y tabaco los que alcanzaron la mayor eficacia (40,18\%). Siendo el tratamiento químico el que alcanzó la menor eficacia con un 35,29\% (Figura 1). Un 1 día después de la tercera aplicación (F2) se determinó que el tratamiento clorpirifós y el insecticida natural a base de ají fueron los más eficientes, con $80 \%$ de eficacia. El insecticida natural a base de tabaco fue el que obtuvo la menor eficacia con un $68,89 \%$. Al analizar la eficacia de la tercera aplicación después de 4 días se observó que la mayor eficacia se obtuvo en el tratamiento químico, con 50\%. Los insecticidas naturales a base de ají y tabaco presentaron eficacia de 20 y $25 \%$ respectivamente (Figura 1). Para la cuarta aplicación (F3), el insecticida a base de tabaco presentó 76,74\% de eficacia, siendo la mayor. El insecticida a base de ají y el control químico presentaron la menor eficacia con 60,47 y $58,14 \%$; respectivamente. A los 4 días después de realizar la aplicación se determinó que el insecticida natural a base de tabaco alcanzó la mayor eficacia con el 54,55\%, mientras que el control químico y el insecticida a base de ají mostraron la menor eficacia con 50,00 y 40,91\% respectivamente (Figura 1).

\section{Loritos verdes (Empoasca kraemeri Ross y Moore)}

La mayor eficacia de los tratamientos sobre la población de loritos verdes 1 día después de la segunda aplicación la obtuvieron los insecticidas a base de ají y tabaco con 41,18\%. Obteniendo la menor eficacia el control químico con un 35,21\%. Cuatro días después de la aplicación, el tratamiento con mayor eficacia fue el control químico con $52,94 \%$ obteniendo la menor eficacia los insecticidas naturales a base de ají y tabaco con 45,45 y $27,27 \%$, respectivamente (Figura 2). Al evaluar la eficacia de los insecticidas naturales 1 día después de la tercera aplicación el control químico fue más eficaz con el 50\%. Obteniendo las menores eficacia los insecticidas a base de ají y tabaco con 20 y $25 \%$, respectivamente. Una vez transcurridas los 4 días, el control químico fue más eficaz con un $52,94 \%$, mientras los insecticidas a base de ají y tabaco obtuvieron las menores eficacia con $0,00 \mathrm{y}$ $11,76 \%$, respectivamente (Figura 2). Al evaluar la eficacia de la cuarta aplicación para 1 día después el insecticida a base de tabaco con $54,55 \%$, fue superior al control químico y al insecticida a base de ají con 50 y 40,91, respectivamente. Una vez transcurrido los 4 días se observó que el tabaco con $38,89 \%$ fue superior al control químico y al ají que presentaron un promedio de $-5,56$ y 22,22, respectivamente (Figura 2).

\section{Variables productivas}

En todas las variables productivas se registró un comportamiento estadístico diferenciado (Tabla 4). El insecticida que mostró superioridad estadística para número de vainas por planta, rendimiento por planta y rendimiento por hectárea fue en insecticida a base de ají. No difiriendo estadísticamente las variables número de granos por vaina y peso de 100 semillas. Para la frecuencia de aplicación no se encontraron diferencias estadísticas en las variables productivas (Tabla 4). Los valores máximos y mínimos obtenidos para las variables productivas

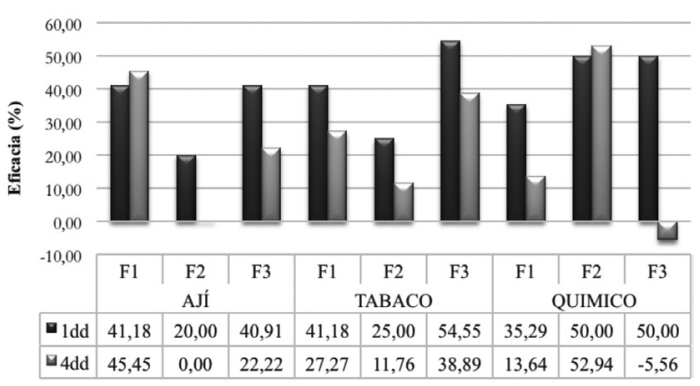

Figura 2. Eficacia de 9 tratamientos en los muestreos efectuados 1 día después y 4 días después de cada frecuencia de aplicación, sobre la población de loritos verdes (Empoasca kraemeri Ross y Moore). 
Tabla 4. Efectos simples y promedios de las variables productivas.

\begin{tabular}{|c|c|c|c|c|}
\hline Tratamiento & $\begin{array}{l}\text { Vainas por planta } \\
\qquad\left(\mathrm{N}^{\circ}\right)\end{array}$ & $\begin{array}{l}\text { Granos por vaina } \\
\qquad\left(\mathrm{N}^{\circ}\right)\end{array}$ & $\begin{array}{c}\text { Peso } 100 \text { semillas } \\
(\mathrm{g})\end{array}$ & $\begin{array}{l}\text { Rendimiento por hectárea } \\
\qquad(\mathrm{kg})\end{array}$ \\
\hline Ají & $7,43 \mathrm{a}$ & $3,60 \mathrm{a}$ & $85,80 \mathrm{a}$ & $1.515,87 \mathrm{a}$ \\
\hline Tabaco & $6,43 \mathrm{~b}$ & $3,45 \mathrm{a}$ & $84,30 \mathrm{a}$ & $1.279,21 \mathrm{~b}$ \\
\hline \multicolumn{5}{|l|}{ Frecuencias (F) } \\
\hline $\mathrm{A} 1=2$ aplicaciones & $6,80 \mathrm{a}$ & $3,50 \mathrm{a}$ & $84,80 \mathrm{a}$ & $1.339,28 \mathrm{a}$ \\
\hline $\mathrm{A} 2=3$ aplicaciones & $7,05 \mathrm{a}$ & $3,50 \mathrm{a}$ & 86,10 a & $1.434,85 \mathrm{a}$ \\
\hline A $3=4$ aplicaciones & $7,00 \mathrm{a}$ & $3,53 \mathrm{a}$ & $84,30 \mathrm{a}$ & $1.418,49 \mathrm{a}$ \\
\hline \multicolumn{5}{|l|}{ Tratamientos } \\
\hline T0 Testigo absoluto & $5,60 \mathrm{c}$ & $3,30 \mathrm{~b}$ & $78,30 \mathrm{~b}$ & $1.091,67 \mathrm{c}$ \\
\hline T1 Químico (clorpirifós) & $7,40 \mathrm{ab}$ & $3,60 \mathrm{a}$ & $86,00 \mathrm{a}$ & $1.462,77 \mathrm{ab}$ \\
\hline T2 Ají + 2 aplicaciones & $7,20 \mathrm{ab}$ & $3,50 \mathrm{ab}$ & $86,20 \mathrm{a}$ & $1.474,92 \mathrm{ab}$ \\
\hline T3 Ají + 3 aplicaciones & $7,90 \mathrm{a}$ & $3,60 \mathrm{a}$ & $86,70 \mathrm{a}$ & $1.567,08 \mathrm{a}$ \\
\hline T4 Ají + 4 aplicaciones & $7,30 \mathrm{ab}$ & $3,60 \mathrm{a}$ & $84,50 \mathrm{ab}$ & $1.505,62 \mathrm{ab}$ \\
\hline T5 Tabaco +2 aplicaciones & $6,40 \mathrm{bc}$ & $3,50 \mathrm{ab}$ & $83,40 a b$ & $1.203,65 \mathrm{c}$ \\
\hline T6 Tabaco +3 aplicaciones & $6,30 \mathrm{bc}$ & $3,40 \mathrm{ab}$ & $85,50 \mathrm{a}$ & $1.302,63 \mathrm{bc}$ \\
\hline T7 Tabaco +4 aplicaciones & $6,70 \mathrm{bc}$ & $3,50 \mathrm{ab}$ & $84,10 \mathrm{ab}$ & $1.331,36 \mathrm{abc}$ \\
\hline C.V. $(\%)$ & 7,08 & $\mathbf{3 , 3 0}$ & 3,16 & 7,51 \\
\hline
\end{tabular}

Promedios con letras iguales dentro de cada factor no presentan diferencias estadísticas según $\mathrm{F}(\mathrm{P}>0,05)$.

estuvieron entre 5,60 a 7,90 para número de vainas por planta, 3,30 a 3,60 para número de granos por vaina, 78,30 a $86,70 \mathrm{~g}$ para el peso de 100 semillas y $1.091,67$ a $1.567,08 \mathrm{~kg} \mathrm{ha}^{-1}$ para el rendimiento. Al respecto, Maggi (2004), sostiene que los extractos de plantas, a más de ser controladores de plagas, también tienen propiedades nutricionales que estimulan los procesos vitales de los cultivos fortaleciéndolos y protegiéndolos de los ataques de las distintas plagas. Asimismo, otros autores han reportado diferencias para el número de vainas por planta de 7,56 a 16,40 (Santos et al., 2009), 7,16 a 8,27 (Shimada et al., 2000) y 10,6 a 10,8 (Abrantes et al., 2011). Igualmente, para el número de granos por vaina de 2,0 a 3,2 (Aguilar et al., 2011), 2,08 a 5,84 (Garcés et al., 2013), 3,7 a 5,1 (Leite et al., 2007), 2,0 a 5,1 (Morales-Rosales et al., 2008), 3,1 a 4,4 (Barrios-Gómez et al., 2010) y 3,9 a 4,6 granos por vaina (Abrantes et al., 2011). Así también, para el peso de 100 semillas 18,9 a 28,7 (Barrios-Gómez et al., 2010), 18 a 23 (Araya y Hernández 2007) y $21 \mathrm{~g}$ (Sueiro et al., 2011). Finalmente, para el rendimiento, valores de 858,2 a 1.459,8 (Folegatti et al., 1999), 1.282 (Barrios y Ortega 1972), 1.556,25 a 3.720,00 kg ha-1 (Garcés, 2011), 1920,5 a 1976,6 (Rodríguez et al., 2007) y 8261471,33 (Tosquy et al., 2007) y $1.734 \mathrm{~kg}$ $\mathrm{ha}^{-1}$ (Araya y Hernández, 2007), la diferencia en cuanto a rendimientos se debe probablemente al uso de distinto material genético, diferentes tipos de insecticidas para el control de insectos-plagas, así como a las condiciones agroclimáticas de los sitios donde se realizaron las investigaciones.

\section{Conclusiones}

El insecticida natural a base de ají tuvo un comportamiento similar al tratamiento químico obteniendo menor población en el ataque de mosca blanca en comparación con el testigo absoluto. Mostrando el mismo comportamiento para el control de lorito verde. En relación a la eficiencia en el control de mosca blanca relacionado a un día después de la aplicación el insecticida natural a base de ají y el tratamiento químico en la aplicación 2 mostraron la mayor eficiencia, en cuanto a los cuatro días después de la aplicación el insecticida a base de tabaco en su aplicación 3 mostró una mayor eficiencia, incluso superando al tratamiento químico en sus tres frecuencias (Figura 1). En cuanto a la eficacia para lorito verde un día después de la aplicación el insecticida natural a base de tabaco en su frecuencia 3 mostró la mayor eficiencia en comparación con los demás tratamientos, mientras que a los cuatro días después de la aplicación fue el tratamiento químico en su aplicación 2 el que mostró mayor eficiencia (Figura 2). La frecuencia 
que mostró los mejores resultados en el control de insectos-plagas fue la frecuencia dos con el insecticida natural a base de ají. Se determinó en relación a las variables productivas que el T3 (ají +3 aplicaciones) mostró los promedios más altos en todas las variables evaluadas.

\section{Literatura Citada}

Abbott, W.S.

1925. A method of computing the effectiveness of an insecticide.

J. Econ. Entomol., 18: 265-267.

Abrantes, F.L., M. de Sá, L.C. Domingues de Souza, P. Pina da Silva, H.M. Simidu, M. Andreotti, S. Buzetti, W.V. VelérioFilho, e N. Arruda.

2011. Uso de regulador de crescimento em cultivares de feijão de inverno. Pesquisa Agropecuária Tropical, 41 (2): 148-154.

Aguilar, G.; Peña, C.; García, J.; Ramírez, P.; Benedicto, S.; Molina, J.

2012. Rendimiento de frijol (Phaseolus vulgaris 1.) en relación con la concentración de vermicompost y déficit de humedad en el sustrato. Agrociencia, 46: 37-50.

Altieri, M.

1999. Bases científicas para una agricultura sustentable Agroecología. Editorial Nordan-Comunidad. Montevideo, Uruguay, $325 \mathrm{p}$.

Altieri, M.; Nicholls, C.

2000. Teoría y práctica para una agricultura sustentable. Red de Formación Ambiental para América Latina y el Caribe. México D.F., México, 250 p.

Araya. R.; Hernández, J.

2007. Variedades de frijol de grano rojo, obtenidas por Fitomejoramiento Participativo en Costa Rica. Programa de Investigación y Transferencia de Tecnología Agropecuaria en frijol (PITTA-Frijol) (en línea) http://www.mag.go.cr/ bibliotecavirtual/a00087.pdf Consultado: 23/Feb/2015.

Barrios, A.; Ortega, O.

1972. Tuy: Nuevo cultivar de frijol (Vigna unguiculata L.). Agronomía Tropical, 25 (2): 103-106. 1975.

Barrios-Gómez, E.J.; López-Castañeda, C.; Kohashi-Shibata,

J.; Acosta-Gallegos, J.; Miranda-Colín, S.; Mayek-Pérez, N.

2010. Rendimiento de semilla, y sus componentes en frijol flor de mayo en el centro de México. Agrociencia, 44 (4): 481-489.

Cardona, C.; Rodríguez, I.; Bueno, J.

2005. Manejo de la mosca blanca o palomilla en los cultivos de habichuela y frijol. Centro Internacional de Agricultura Tropical (CIAT). Cali, Colombia, $31 \mathrm{p}$.

Casanova, H.; Ortiz, C.; Pelbez, C.; Vallejo, A.; Moreno, M.

E., \& Acevedo, M.

(2002). Insecticide formulations based on nicotine oleate stabilized by sodium caseinate. Journal of agricultural and food chemistry, 50, 6389-6394.

Ernest, E.G.; Falconí-Castillo, E.; Peralta-Idrovo E.; Kelly, J. 2008. Encuesta a productores para orientar el fitomejoramiento en frijol en Ecuador. Agronomía Mesoamericana, 19 (1): 07-18.

Firester, $\mathrm{K}$.

2011 Plant Extracts as Natural Insecticides. Pág. 4-5. New York, EE.UU. Disponible en: http://www.amnh.org/learn-teach/ young-naturalist-awards/winning-essays 2/2011-winningessays/plant-extracts-as-natural-insecticides Consulrado el 28/01/2015.
Folegatti, M.; Paz, V.P.; De Oliveira, A.

1999. Rendimento do feijoeiro irrigado submetido a diferentes lâminas de água com irrigação por sulco. Revista Brasileira de Engenharia Agrícola e Ambiental, 3 (3): 281-285.

Fuentes-Contreras, E.; Basoalto, E.; Sandoval, C.; Pavez, P.; Leal, C.; Burgos, R.

2007. Evaluación de la eficacia, efecto residual y de volteo de aplicaciones en pretrasplante de insecticidas nicotinoides y mezclas de nicotinoide-piretroide para el control de Myzus persicae (Hemiptera: Aphididae) en tabaco. Agricultura Técnica. 67 (1): 16-22.

Garcés-Fiallos, F.R.

2011. Modelo de ponto crítico para estimar danos causados pela mela na cultura do feijoeiro. Ciencia y Tecnología, 4 (1): $1-4$.

Garcés, F.; Aguirre A.; Garcés R.; Díaz, E.; Sánchez, F.; Prieto, O. 2013. Diseases and yield components of sixteen bean genotypes in Quevedo, Ecuador. Ciencia y Tecnología, 6 (2): 31-39

Garcés, F

2013. Cuantificación de enfermedades en líneas promisorias y variedades de frejol en Quevedo, Ecuador. Biotecnología en el Sector Agropecuario y Agroindustrial, 11 (1): 196-207.

Garcés, F.O.; Zamora Garcés, R.; Díaz, T.

2015. Potencial agronómico de 18 líneas de frejol F6 en Ecuador. IDESIA, 33 (2): 107-118.

Hall, R.

1994. Compendium of bean diseases. APS Press. St. Paul, MN. USA, $73 \mathrm{p}$.

Horwith, B

1985. A role for intercropping in modern agriculture. BioScience, 35 (5): 286-291.

Instituto Nacional de Estadística y Censos.

2002. III Censo Nacional Agropecuario. Resultados Nacionales y Provinciales. INEC, Ministerio de Agricultura y GanaderíaMAG, Servicio de Servicio y Censo Agropecuario-SICA. Quito, Ecuador, 255 p.

Karlsson, M.F.

2005. Control de mosca blanca (Aleurotrachelus socialis) en yuca (Manihot esculenta). SLU Communications, Swedish University of Agricultural Sciences, Uppsala, Suecia, 78 p.

Leite, U.; Araújo, G.A.; Miranda, G.; Vieira, R.; Carneiro, J.E.; Pires, A.

2007. Rendimento de grãos e componentes de rendimento do feijoeiro em função da aplicação foliar de doses crescentes de molibdênio. Acta Scientiarum Agronomy, 2 (1): 113-120.

Maggi, M.

2004. Insecticidas Naturales. Origen de los pesticidas naturales. Laboratorio de Química Fina y Productos Naturales. Agencia Córdoba Ciencia - Unidad CEPROCOR. Cordoba, Argentina, 8 p.

Manzano. L.; Mejia. L.

1998. Memorias. Desarrollo de los sistemas de producción cebolla de bulbo (Ocañera) y frijol rosado o zaragoza 
mediante una agricultura orgánica libre de agroquímicos. Corporacón Colombiana de Invetigación Agropecuaria. Ocaña, Colombia, 84 p.

Mendoza, J.

1997. Evaluación de extractos vegetales para el control de plagas del frejol (Phaseolus vulgaris L). Revista Ceiba, 38 (1): 90.

Morales-Rosales, E.J.; Escalante-Estrada, J.A.S.; LópezSandoval, J.A.

2008. Crecimiento, índice de cosecha y rendimiento de frijol (Phaseolus vulgaris L.) en unicultivo y asociado con girasol (Helianthus annuus L.). Universidad y Ciencia, 24 (1): 1-10. Murillo, W.; Araque, P.; Pelaéz, C.

2012. Actividad fungicida e insecticida de emulsiones agua/ aceite de mezclas de extractos de Nicotiana tabacum, Azadiractha indica y eucaliptus tereticornis. Información Tecnológica. Vol. 23 (1): 139-152

Peña, M.; Castro, J.; Soto, A.

2013. Evaluación de insecticidas no convencionales para el control de Aphis gossypii Glover (Hemiptera:Aphididae) en fríjol. Rev. U.D.C Act. \& Div. Cient. 16 (1): 131-138.

Pérez, A.; Landero, C.

2009. Agricultura y deterioro ambiental. Elementos, 73 (16) 19-25.

Reyes, E.

2008. History, nature and bean's nutritious quality. Investigación Científica, 4 (3): 1-21.

Rodríguez, J.; López, E.; Tosquy, O.

2007. Componentes de rendimiento del frijol negro en diferentes fechas de siembra durante el ciclo otoño-invierno en el norte de Veracruz, México. Revista Científica Biológico Agropecuaria Tuxpan, 2 (3): 266-270.

Santos, J.F.; Tavares-Grangeiro, J.I.; de Brito, C.H.; AlmeidaSantos, M. do C.C.

2009. Produção e componentes produtivos de variedades de feijão caupi na microregião Cariri Paraibano. Engenharia Ambiental, 6 (1): 214-222.

Shimada, M.M.; Arf O.; De Sá, M.E.

2000. Componentes do rendimento e desenvolvimento do feijoeiro de porte ereto sob diferentes densidades populacionais. Bragantia, 59 (2): 181-187.

Sueiro, A.; Rodríguez, M.; De la Cruz, S.

2011. El uso de biofertilizantes en el cultivo del frijol: una Alternativa para la agricultura sostenible en Sagua la Grande. Observatorio de la Economía Latinoamericana, 159: 3-7.

Tosquy, O.; Esqueda, V.; Zetina, R.; Durán, A.; Rodríguez, J. 2007. Efecto de diferentes sistemas de fertilización en el rendimiento de frijol de humedad residual en Veracruz. Revista Científica Biológico Agropecuaria Tuxpan, 2 (3): 289-293.

Valladolid, J.; Pantaleón, S.; Castillo, O.; Aquino, J.

1998. Producción de Menestras de Exportación para Agricultores. Producción de Leguminosas de grano para exportación. México, pp. 10-25.

Voysest, V.

2000. Mejoramiento genético del fríjol (Phaseolus vulgaris L.). Centro Americano de Agricultura Tropical. Cali, Colombia, $195 \mathrm{p}$. 
